\title{
ESTIMATING MATCHING DISTANCE BETWEEN SPECTRA
}

\section{ABDELKRIM NOKRANE}

Abstract. We show that if $a, b$ are elements of an unital Banach algebra such that almost all convex combinations of $a$ and $b$ have a finite spectrum of cardinality $n$, then the optimal matching distance between their spectra satisfies

$$
D(\sigma(a), \sigma(b)) \leqslant c_{n}(\|a\|+\|b\|)^{1-1 / n}\|a-b\|^{1 / n},
$$

where $c_{n} \leqslant 8(1+1 / n)(n / 2)^{1 / n}$.

Mathematics subject classification (2000): 15A42, 47A10.

Keywords and phrases: spectrum, algebroid multifunction, matching distance.

\section{REFERENCES}

[1] B. Aupetit, A Primer on Spectral Theory, Springer, 1991.

[2] R. Bhatia, Matrix Analysis, Springer, 1997.

[3] R. Bhatia, L. Elsner And G. Krause, Bounds for the Variation of the Roots of a Polynomial and the Eigenvalues of a Matrix, Linear Algebra Appl., 142 (1990), 195-209.

[4] R. Bhatia AND D. DRISSI, Perturbation theorems for Hermitian elements in Banach algebras, Studia Math., 134, 2 (1999), 111-117.

[5] M. Brešar And P. ŠEmrl, Derivation mapping into the socle, Math. Proc. Camb. Phil. Soc., 120 (1996), 339-346.

[6] Y. Chen, A. NoKRANE AND T. RANSFORD, Estimates for the spectrum near algebraic elements, Linear Algebra Appl., 308 (2000), 153-161.

[7] L. ELSNER, An optimal bound for the spectral variation of two matrices, Linear Algebra Appl., 71 (1985), 77-80.

[8] G. KRAUSE, Bounds for the variation of matrix eigenvalues and polynomial roots, Linear Algebra Appl., 208/209 (1994), 73-82.

[9] A. Nokrane And T. Ransford, Schwarz's Lemma for Algebroid Multifunctions, Complex Variables Theory Appl., 45 (2001), 183-196.

[10] T. Ransford, Potential Theory in the Complex Plane, London Mathematical Society, Cambridge University Press, 1995. 